\title{
Neonatal chlamydial eye infection: an epidemiological and clinical study
}

\author{
KENNETH PERSSON, ${ }^{1}$ ROLF RÖNNERSTAM,${ }^{2}$ LARS SVANBERG, ${ }^{3}$ AND \\ MADLI-ANN POHLA ${ }^{2}$
}

From the Departments of ${ }^{1}$ Clinical Virology, ${ }^{2}$ Ophthalmology, and ${ }^{3}$ Obstetrics and Gynaecology, University of Lund, Malmö General Hospital, Malmö, Sweden

SUMmaRY Neonatal chlamydial eye infection was detected in 39 cases of 281 infants with purulent conjunctivitis seen at the Department of Ophthalmology. Most cases of neonatal chlamydial eye infection (90\%) were detected during the first month of life, and no case was found after 2 months of age. C. trachomatis was not isolated from any of 161 healthy control infants at 3-4 weeks of age. Genital chlamydial infection was demonstrated in $50 \%$ of the mothers to infected infants. Two of these women developed post-partum complications possibly due to chlamydial infection. Mothers of infected infants tended to be younger than average. Of 23 cases examined 2 years later, late sequelae were identified in 3.

Chlamydia trachomatis was first isolated from the eyes of an infant with neonatal conjunctivitis in $1959 .^{1}$ The organism could also be recovered from the uterine cervix of the mother of an infected infant. Inoculation of volunteers with the isolated agent caused clinical signs similar to acute trachoma with typical cellular inclusions. ${ }^{2}$ Inclusion conjunctivitis in newborn babies has been known since the first decade of this century ${ }^{34}$ and was called inclusion blenorrhea by Lindner. ${ }^{5}$

It is now recognised that $C$. trachomatis is one of the major causes of sexually transmitted diseases in industrialised countries. The infection can be transmitted to the infant at delivery when the mother is affected. The genital strains of $C$. trachomatis belong with few exceptions to one of the serogroups D through K. Classical trachoma in developing countries is associated with ocular strains generally belonging to the serotypes $\mathrm{A}$ through $\mathrm{C}$. Neonatal chlamydial eye infection has been considered virtually nonexistent in areas with endemic trachoma like Egypt and Saudi Arabia. In Africa both neonatal chlamydial eye infection and classical trachoma have been observed in the same population, ${ }^{6}$ and recent reports indicate that neonatal chlamydial infection may be a substantial problem in different African countries. $^{78}$

Correspondence to Kenneth Persson, MD, Department of Clinical Virology, Malmö General Hospital, S-214 01 Malmö, Sweden.
Prospective studies during the last few years in the USA have shown that $2-18 \%$ of pregnant women have genital chlamydial infection and that $20-35 \%$ of the infants of infected mothers develop symptomatic eye infection..$^{9-13}$ A smaller proportion of infants present a pneumonia syndrome at 1-3 months of age with potentially a life-threatening severity. Some two-thirds of all infants exposed to the infection have serological signs of chlamydial infection. Infants to mothers with chlamydial infection were also far more likely to contract the infection than control children of mothers in whom infection could not be demonstrated.

Genital chlamydial infection in puerperal women in Sweden has been detected in $2 \%$ and $7 \%$ in different areas. ${ }^{1415}$ In this study the occurrence of $C$. trachomatis in children with neonatal conjunctivitis and in healthy infants was compared. The late consequences of chlamydial conjunctivitis were evaluated. Maternal infections were also studied as well as the outcome of the pregnancies.

\section{Materials and methods}

Study group. Infants below 6 months of age attending the Department of Ophthalmology for purulent eye inflammation were examined for the presence of C. trachomatis in the eyes. However, all infants fulfilling these criteria could not be examined for 
chlamydial infection for practical reasons, but there was no obvious bias in the group of infants examined. This group included 281 infants. All infants received Credé's prophylaxis by silver nitrate. There is only one hospital serving a city population of a quarter of a million people. Patients with acute symptoms of some significance are normally seen at the hospital.

When a positive chlamydial isolation was obtained from the eyes of an infant, the mother was referred to a gynaecological examination for detection of genital chlamydial infection.

Follow-up examination was possible on 23 infants with chlamydial eye infection. It was performed when the infants were 6 months to 3 years and 11 months old, with a mean age in the group of $2 \cdot 1$ years.

Control group. 161 healthy children 3-4 weeks of age were examined for the presence of chlamydial eye infection at a routine examination at health care centres for children, where all infants are seen at regular intervals. Both sexes were equally represented.

Chlamydial isolation. Specimens for chlamydial isolation were obtained by cotton-tipped swabs and transported in 2 SP-medium. Irradiated McCoy cell cultures were inoculated in duplicate and chlamydial inclusions detected after $72 \mathrm{~h}$ by iodine staining.

\section{Results}

Neonatal chlamydial eye infection. During a 4-year period, 1978 through 1981, 39 cases of ophthalmia neonatorum associated with Chlamydia trachomatis were detected among 281 infants with conjunctivitis. Most chlamydial infections were detected before the infants reached 1 month of age, and after 2 months of age no more chlamydial isolations were obtained (Table 1). In 36 cases the infants were delivered at the Department of Obstetrics at this hospital, and in 3 cases the infants were born at other hospitals but the parents moved to Malmö soon after delivery. As seen in Table 2 on average 9 out of $2400(0.4 \%)$ infants born each year with neonatal chlamydial infection were found. Delivery records were available in 34 cases of the infants with chlamydial conjunctivitis. These 34 infants were born after 34 to 42 gestational weeks, with a mean at 40.0 weeks, and weighed between 2250 and $4110 \mathrm{~g}$, mean weight $3290 \mathrm{~g}$. At delivery the infants who developed chlamydial infection were assessed for vital signs and assigned an Apgar score of 8 through 10 with a mean of 9 . Only one infant was born prematurely; the other 33 deliveries were considered normal, although vacuum extraction was performed in one case. Among the infants with chlamydial eye infection none was delivered by caesarean section, though the average frequency of caesarean section at this hospital is about $10 \%$. A third of the infants infected were boys,
Table 1 Detection of chlamydial eye infection at different ages

\begin{tabular}{llr}
\hline Age & \multicolumn{2}{l}{ Chlamydial cases } \\
\cline { 2 - 3 } & No. examined & $\%$ \\
\hline$<1$ month & $35 / 131$ & 27 \\
$1-2$ months & $4 / 56$ & 7 \\
$2-6$ months & $0 / 94$ & 0 \\
Total & $39 / 281$ & 14 \\
\hline
\end{tabular}

Table 2 Infants with chlamydial eye infection of liveborn children each year in Malmö

\begin{tabular}{lll}
\hline & $\begin{array}{l}\text { Cases with chlamydial } \\
\text { conjunctivitis }\end{array}$ & Liveborn infants \\
\hline 1978 & 7 & 2401 \\
1979 & 9 & 2397 \\
1980 & 8 & 2358 \\
1981 & 12 & 2391 \\
Total & $36(0 \cdot 4 \%)$ & 9547 \\
\hline
\end{tabular}

but the difference between boys and girls among affected infants was not statistically significant. The whole group of children consisted of girls and boys in equal proportions.

When they were seen in the hospital, infants with chlamydial eye infection usually presented with moderate to severe symptoms. These were often more pronounced in one eye, especially in the early stages of the illness. There was often substantial swelling of the eyelids, conjunctival congestion, mucopurulent discharge, and in some advanced cases a follicular roughness was seen in the fornices. Pseudomembrane formation was also detected in some cases.

Control infants. A control group consisting of 161 healthy infants were examined for the presence of chlamydial eye infection at 3 to 4 weeks of age when chlamydial conjunctivitis is usually manifest. In no case was $C$. trachomatis demonstrated in conjunctival swabs.

Maternal chlamydial infection. A gynaecological

Table 3 Age distribution of mothers to infants with chlamydial eye infection born in Malmö

\begin{tabular}{lcc}
\hline $\begin{array}{l}\text { Age of mothers } \\
\text { (years) }\end{array}$ & $\begin{array}{l}\text { Mothers with infected } \\
\text { infants/total number of } \\
\text { childbearing women } \times 10^{-3}\end{array}$ & $\%$ \\
\hline$<19$ & $4 / 0 \cdot 4$ & $1 \cdot 0$ \\
$20-24$ & $20 / 2 \cdot 5$ & $0 \cdot 8$ \\
$25-29$ & $8 / 3 \cdot 6$ & $0 \cdot 2$ \\
$30-34$ & $3 / 2 \cdot 1$ & $0 \cdot 1$ \\
$>35$ & $1 / 0 \cdot 8$ & $0 \cdot 1$ \\
Total & $36 / 9 \cdot 4$ & $0 \cdot 4$ \\
\hline
\end{tabular}


examination was arranged for mothers to the infected infants. Specimens for isolation of chlamydia were obtained from 33 of the women. C. trachomatis was isolated in $16(48 \%)$ cases. Post-partum endometritis developed in one case and salpingitis in another. These 2 women received antibiotic treatment before chlamydial isolation was attempted. The age distribution of the mothers with infected children was different from that of the whole group of mothers (Table 3). Mothers with infected children tended to be younger than average, 24 years for mothers with infected infants and 28 for the whole group. There was no difference in age between the 16 mothers with detectable chlamydial infection (mean age 23.4 years) and the 17 women in whom C. trachomatis was not demonstrated even though their children had the infection (mean age $24 \cdot 2$ years, $t=0 \cdot 61$, NS).

Treatment. After $C$. trachomatis was found in eye swabs from the infants, erythromycin ethylsuccinate, 40-50 mg per $\mathrm{kg}$ body weight per day, was given by mouth for 2 weeks. Topical chloramphenicol or sulphamethizole were also used regularly to combat possible coinfection by other pathogens. In some cases symptoms had persisted for several days despite topical treatment, but a prompt response was seen when systemic treatment was instituted. One case was still chlamydia-positive after one week of erythromycin treatment but healed microbiologically and clinically after another week. Chlamydial isolation attempted from the conjunctivae of 20 cases after systemic erythromycin treatment was negative in all cases. Infected mothers were given erythromycin stearate, $500 \mathrm{mg}$ twice a day, for 2 weeks.

Follow-up examination. Of the 39 infants with neonatal chlamydial infection 23 could be traced for a late follow-up examination, which was performed when the children had reached the age of $2 \cdot 1$ years on average. Swabs from the eyes were obtained from 22 cases and were uniformly negative for $C$. trachomatis. Late sequelae were discovered in 3 cases. Two infants had tarsal scars and one had corneal lesions consisting of superficial maculae but no signs of activity. In 2 of these cases systemic treatment intended to control chlamydial infection had been delayed, and lack of compliance was admitted in one case. It was not possible to examine the smallest children by the corneal microscope, so that minor lesions may have remained undetected in some cases.

\section{Discussion}

In this study $C$. trachomatis was detected in the eyes of infants with purulent conjunctivitis attending the Department of Ophthalmology in $27 \%$ of the cases during the first month of life and in $7 \%$ of the infants between 1 and 2 months old. No further cases were discovered thereafter. Among 161 healthy control children chlamydial eye infection could not be demonstrated in any case. These control infants were examined at 3-4 weeks of age, as it was known from an earlier study ${ }^{15}$ that $C$. trachomatis could usually not be detected at 4-5 days of age, although chlamydial infection may be demonstrated later on. The presence of $C$. trachomatis in the eyes was therefore associated with symptoms in a highly significant way. At the time of diagnosis by isolation of the organisms, as stated in this study, the infants were indisputably infected, but symptoms had usually been present for some days to weeks before. The incubation time for chlamydial eye infection in neonates has been estimated to be between 3 and 19 days on the assumption that the infants are infected at birth. The distribution of cases observed in this study indicated that the infants were infected at about the same time early in life, probably at birth, and that there were no signs of continuous dissemination of chlamydial infection among infants up to 6 months of age. Therefore it seems likely that the mothers were the source of infection despite the fact that C. trachomatis could be demonstrated in only $50 \%$ of mothers of infected infants. These negative results may be due to technical inadequacies. It is also possible that the infection had cleared up by the time the isolation swabs were taken some weeks after delivery. Although nosocomial infection of infants may not be impossible, its occurrence has not been reported to our knowledge.

The incidence of neonatal chlamydial eye infections deduced from the clinical cases of conjunctivitis amounts to on average 4 per 1000 live born infants a year. This figure coincides with the frequency found in a prospective study. ${ }^{15}$ Mild cases may not have been seen at the hospital, and on the assumption of an attack rate of 20-35\% of clinical conjunctivitis among infants exposed to risk the expected frequency in our population would be $0.5-0.8 \%$, indicating that probably more than $50 \%$ of the cases were detected. The frequency of asymptomatic carriers should be less than $1 / 161$.

Gonococcal infection in pregnancy is now uncommon in our population, and Credé's prophylaxis by silver nitrate instillation in the eyes of all neonates is currently being abandoned in this country. Neonatal chlamydial eye infections, however, have not been generally recognised until recently. The detection of a sexually transmitted agent in a neonate indicates a maternal and possibly also paternal genital infection by the same agent. In our study we detected one case of salpingitis and one case of endometritis post partum among mothers to infants with chlamydial infection. Although a causal relationship could not be established between $C$. trachomatis and 
clinical symptoms in these cases, a definite increase in puerperal morbidity has been associated with chlamydial infection. ${ }^{16}$

It must be assumed that chlamydial conjunctivitis in neonates existed in our population before the last few years; when a definite diagnosis by isolation of the agent has become possible. The general experiences of ophthalmologists suggest that neonatal conjunctivitis heals sooner or later after topical treatment, and it has been known since the condition was first identified at the beginning of the century that inclusion blennorrhea is a self-limiting disease. It was also noticed then that scars developed in a proportion of cases. ${ }^{5}$ Higher standards of living have not changed the situation, and late sequelae after chlamydial conjunctivitis have been repeatedly reported in recent studies. ${ }^{17-21}$ Chronic relapsing infection may also occur. ${ }^{22}$ In our study 2 patients had tarsal scars and one child corneal lesions at late follow-up out of the 23 children who were examined. There was no evidence of active infection, and all children had negative chlamydial isolations. It was discovered that treatment with oral erythromycin had either been delayed or was very inadequate in the 3 cases with late sequelae. This is not to say, however, that lesions would not have developed had treatment been optimal. But the risk of late sequelae is likely to rise if inflammation is intense or prolonged. Both these factors could be restricted by early effective treatment.

Oral treatment must be considered the best, as neonatal chlamydial infections are usually multifocal, involving the conjunctivae, nasopharynx, the lungs, the intestinal tract, and the vagina. ${ }^{23}$ Recently there has been some concern about the efficacy of erythromycin for the eradication of neonatal infection by $C$. trachomatis, because the organism could be isolated again from the eyes or nasopharynx in a substantial proportion of cases after treatment. ${ }^{24}{ }^{25}$ These treatment failures may be due to lack of the patient's compliance, low absorption, or inability to tolerate the drug. Decreased sensitivity of C. trachomatis to erythromycin has also been observed in some strains. ${ }^{26}$ None of our 23 patients had signs of active infection at late foilow-up, and isolations from 22 of these patients were negative. Eye swabs were available from 20 cases after treatment when the acute infection had subsided. Chlamydia could not be recovered from any of these specimens.

Our study shows that neonatal chlamydial eye infection constituted a substantial proportion of the cases with purulent conjunctivitis seen at the Department of Ophthalmology. The inflammatory reaction was sometimes very intense, with pseudomembrane formation. A lingering course was the rule until the proper diagnosis was reached and oral treatment instituted.

Late sequelae were detected in some cases. Chlamydial infection in the neonate indicates infection in the parents by the same agent, which necessitates close co-operation between ophthalmologist, paediatrician, gynaecologist, and venereologist in handling these cases. Although laboratory facilities are generally inadequate to support a preventive programme, the screening of pregnant women in the youngest age groups would certainly be economically justified.

\section{References}

1 Jones BR, Collier LH, Smith CH. Isolation of virus from inclusion blennorrhea. Lancet 1959; i: 902-5.

2 Jones BR, Collier LM. Inoculation of man with inclusion blennorrhea virus. Ann NY Acad Sci 1962; 98: 212-26.

3 Stargardt $K$. Epithelzellveränderungen beim Trachom und anderen Conjunktivalerkrankungen. Albrecht von Graefes Arch Klin Ophthalmol 1909; 69: 525-42.

4 Halberstädter L, von Prowazek S. Über Chlamydozoenbefunde bei Blennorrhea neonatorum non gonorrhoica. Berl Klin Wochenschr 1909; 46: 1839-40.

5 Lindner K. Gonoblennorrhöe, Einschlussblennorrhöe und Trachom. Albrecht von Graefes Arch Klin Ophthalmol 1911; 78: 345-80.

6 Sowa S, Sowa J, Collier LH. Investigation of neonatal conjunctivitis in the Gambia. Lancet 1968; ii: 243-7.

7 Mabey DCW, Whittle HC. Genital and neonatal chlamydial infection in a trachoma endemic area. Lancet 1982; ii: 300-1.

8 Meheus A, Delgadillo Widi-Wirsky R, Piot P. Chlamydial ophthalmia neonatorum in central Africa. Lancet 1982; ii: 882.

9 Chandler JW, Alexander ER, Pheiffer TA, Wang S-P, Holmes KK, English M. Ophthalmia neonatorum associated with maternal chlamydial infections. Trans Am Acad Ophthalmol Otolaryngol 1977; 83: 302-8.

10 Frommell GT, Rothenberg R, Wang S-P, McIntosh K. Chlamydial infection of mothers and their infants. J Pediatr 1979; 95: 28-32.

11 Hammerschlag MR, Anderka M, Semine DZ, McComb D, McCormack WM. Prospective study of maternal and infantile infection with Chlamydia trachomatis. Pediatrics 1979; 64: 142-8.

12 Schachter J, Holt J, Goodner E, Grossman M, Sweet R, Mills J. Prospective study of chlamydial infection in neonates. Lancet 1979; ii: $377-80$.

13 Heggie AD, Lumicao GG, Stuart LA, Gyves MT. Chlamydia trachomatis infection in mothers and infants. Am J Dis Child 1981; 135: 507-11.

14 Mårdh P-A, Helin I, Bobeck S, Laurin J, Nilsson T. Colonisation of pregnant and puerperal women and neonates with Chlamydia trachomatis. Br J Vener Dis 1980; 56: 96-100.

15 Persson K, Rönnerstam R, Svanberg L, Holmberg L. Maternal and infantile infection with chlamydia in a Swedish population. Acta Paediatr Scand 1981; 70: 101-5.

16 Wager GP, Martin DH, Koutsky L, et al. Puerperal infectious morbidity: relationships to route of delivery and to antepartum Chlamydia trachomatis infection. Am J Obstet Gynecol 1980; 138: 1028-33.

17 Freedman A, Al-Hussaini MK, Dunlop EM, et al. Infection by TRIC agent and other members of the Bedsonia group, with a note on Reiter's disease. II Ophthalmia neonatorum due to TRIC agent. Trans Ophthalmol Soc UK 1966; 86: 313-20.

18 Watson PG, Gairdner D. TRIC agent as a cause of neonatal eye sepsis. Br Med J 1968; iii: 527-8. 
19 Forster RK. Dawson CR. Schachter J. Late follow-up of patients with neonatal inclusion conjunctivitis. Am J Ophthalmol 1970; 69: 467-72.

20 Mordhorst $\mathrm{CH}$. Dawson C. Sequelae of neonatal inclusion conjunctivitis and associated disease in parents. Am J Ophthalmol 1971; 71: 861-7.

21 Goscienski PJ, Sexton RR. Follow-up studies in neonatal inclusion conjunctivitis. Am J Dis Child 1972; 124: 180-2.

22 Mordhorst CH, Wang S-P. Grayston JT. Childhood trachoma in a nonendemic area. Danish trachoma patients and their close contacts, 1963 to 1973 . JAMA 1978; 239: 1765-71.

23 Schachter J, Grossman M, Holt J, Sweet R, Spector S. Infection with Chlamydia trachomatis: involvement of multiple anatomic sites in neonates. J Infect Dis 1979; 139: 232-34.

24 Rees E, Tait IA, Hobson D, Karayiannis P, Lee N. Persistence of chlamydial infection after treatment for neonatal conjunctivitis. Arch Dis Child 1981; 56: 193-8.

25 Sandström KI, Bell TA, Chandler JW, et al. Diagnosis of neonatal purulent conjunctivitis caused by Chlamydia trachomatis and other organisms. In: Mårdh P-A, et al., eds. Chlamydia infections. Amsterdam: Elsevier, 1982: 217-20.

26 Mourad A, Sweet RL. Sugg N, Schachter J. Relative resistance to erythromycin in Chlamydia trachomatis. Antimicrob Agents Chemother 1980; 18: 696-8. 